\title{
Pedicle of Vertebral Arch
}

National Cancer Institute

\section{Source}

National Cancer Institute. Pedicle of Vertebral Arch. NCI Thesaurus. Code C96274.

A thick process on either side of the vertebral body that connects the latter to the vertebral arch. 